\title{
Citrus greening in Jamaica
}

Among several events during the past two months, I would like to highlight the spread of the citrus greening disease in Jamaica. This information has just been confirmed by the Ministry of Agriculture of this country, indicating that, if not properly managed, this disease could destroy the citrus business, estimated at 3.7 billion U.S. \$.

This new epidemic could strongly affect citrus production in this country, one of the oldest in the Western world, in addition to the crisis it faced after the spread of the Citrus Tristeza Virus in the 1990s.

Jamaica is an example that illustrates the economic and social impact that such diseases could have without any preventive measures or risk anticipation.

After Brazil, Florida and California, and now Jamaica, we know that the risk is very high for the Mediterranean region, where the citrus industry is a key element of the economy of these countries.

In 2007, I already stressed the importance of such a risk and pleaded for real research programmes making it possible to prevent such a plague, while being based on scientific arguments which make it possible to reconcile effectiveness and production with safety and respect for the environment.

Very little has been done since then, as if the risk was non-existent.

Generally, in international fora or conferences, in the media, in research institutions, and in the minds of donors, emerging diseases mainly mean human and animal diseases, probably because there is a direct risk for ourselves. Emerging plant diseases are less considered, whereas they could have a very strong economic and social impact.

It is time to change our mindset and to think that plant pests and diseases could have devastating effects. The strong emphasis put on human and animal diseases must not hide the threat related to plant species.

Dr. Jacky Ganry Chairman of the Section on Tropical and Subtropical Fruits ISHS 


\section{Le greening des agrumes en Jamaïque}

Parmi les événements qui ont marqué les deux dernier mois, je voudrais mentionner tout particulièrement l'arrivée du greening des agrumes en Jamaique. Cette information vient juste d'être confirmée par le ministère de l'Agriculture de ce pays, indiquant que, faute d'être correctement contrôlée, cette maladie pourrait mettre en péril l'activité agrumicole estimée à 3,7 milliards de \$US.

Le greening pourrait affecter fortement la production agrumicole de ce pays, l'une des plus anciennes du monde occidental, qui avait déjà subi une crise après la propagation du virus de la Tristeza dans les années quatre-vingt-dix.

La Jamaique est un exemple qui illustre l'impact économique et social que de telles maladies dévastatrices pourraient avoir faute de mesures préventives et d'anticipation du risque.

Après le Brésil, la Floride et la Californie et, maintenant, la Jamaïque, nous savons que le risque est très haut pour la région méditerranéenne où la production agrumicole est un élément clé de l'économie des pays.

En 2007, j'avais déjà souligné l'importance d'un tel risque et plaidé pour que soient développés de véritables programmes de recherche permettant de prévenir un tel fléau en s'appuyant sur des bases scientifiques conciliant efficacité et production avec sureté et respect de l'environnement.

Très peu a été fait depuis lors comme si le risque était inexistant.

D'une façon générale, dans les conférences ou forums internationaux, dans les médias, dans les instituts de recherche, dans l'esprit des donateurs, ce sont surtout les maladies humaines et animales qui sont considérées comme maladies émergentes, probablement parce qu'il y a un risque direct pour nous-mêmes. Les maladies végétales ont tendance à être oubliées alors qu'elles peuvent avoir un impact économique et social très fort.

Il est temps de changer notre mentalité et de réaliser que les maladies végétales peuvent avoir des effets dévastateurs. L'importance accordée aux maladies humaines et animales ne doit pas faire oublier les menaces qu'elles représentent sur les espèces végétales.

Dr. Jacky Ganry

Président de la section Fruits tropicaux et subtropicaux ISHS 